\title{
Normal-to-incommensurate phase transition in the spin-Peierls compound TiOCl: An x-ray diffraction study
}

\author{
Andreas Schönleber, Galina Shcheka, and Sander van Smaalen* \\ Laboratory of Crystallography, University of Bayreuth, Bayreuth, Germany \\ (Received 31 July 2007; revised manuscript received 25 January 2008; published 14 March 2008)
}

\begin{abstract}
$\mathrm{X}$-ray diffraction on $\mathrm{TiOCl}$ indicates a temperature-independent $\mathbf{c}$-axis unique monoclinic distortion of the crystal lattice of $\gamma=90.023(2)^{\circ}$ within the incommensurate phase between $T_{c 1}=67 \mathrm{~K}$ and $T_{c 2}=90 \mathrm{~K}$. The transition at $T_{c 2}$ is described as a first-order phase transition driven by the formation of antiferromagnetic order on the Ti-O bilayers that is governed by the monoclinic lattice distortion. The temperature-dependent incommensurate modulation is considered to represent the growth of the number of spin-Peierls-type singlet pairs on cooling. At $T_{c 1}$, too many electrons have thus been removed from the antiferromagnetic state for it to be stable, and the crystal transforms into the spin-Peierls state.
\end{abstract}

DOI: 10.1103/PhysRevB.77.094117

PACS number(s): 75.30.Et, 61.50.Ks, 61.66.Fn, 75.30.Kz

\section{INTRODUCTION}

$\mathrm{TiOCl}$ and $\mathrm{TiOBr}$ have attracted attention as onedimensional (1D) $S=\frac{1}{2}$ spin-chain compounds that are in a dimerized, spin-Peierls state at low temperatures. ${ }^{1-5}$ Both compounds exhibit similar complicated phase diagrams, with, on cooling, a phase transition at $T_{c 2}$ toward an incommensurately modulated state, followed by a first-order phase transition at $T_{c 1}$ toward the spin-Peierls state. ${ }^{1-16}$ Transition temperatures are $T_{c 1}=67.4 \mathrm{~K}$ and $T_{c 2}=90 \mathrm{~K}$ for $\mathrm{TiOCl}$, while lower transition temperatures have been found for TiOBr. ${ }^{1,2,8} \mathrm{TiOCl}$ and $\mathrm{TiOBr}$ crystallize in the FeOCl structure type with the orthorhombic space group Pmmn and lattice parameters $a=3.778, b=3.355$, and $c=8.027 \AA$ for $\mathrm{TiOCl}$ at room temperature. ${ }^{17}$

Diffraction studies and crystal structures may guide the development of microscopic models explaining physical properties and phase diagrams, because the observed structural distortions point at particular interactions between atoms, while they can exclude other models. ${ }^{2,3,6,9,14,18,19}$ However, determination of the structural distortions is often not straightforward, because of the possibility of loss of point symmetry at a phase transition, while the formation of a supercell is unambiguously indicated by the superlattice reflections.

The problem arises due to the fact that the loss of point symmetry is almost invariably accompanied with the development of a domain structure comprising all possible orientations of the low-symmetry structure on the high-symmetry lattice. The apparent diffraction symmetry of such a twinned crystal then is equal to the diffraction symmetry in the highsymmetry case. Lattice distortions are often small, such that any splittings of overlapping Bragg reflections can only be measured in high-resolution diffraction experiments. Synchrotron radiation provides the required resolution. However, all crystals of $\mathrm{TiOCl}$ and $\mathrm{TiOBr}$ that we have been able to grow are of less than optimal quality, such that the width of Bragg reflections for reorientations of the crystal ( $\omega$ scans) is limited by the mosaic spread of the material to approximately $0.1^{\circ}$ - sufficiently wide to hide the monoclinic splitting. A splitting of Bragg reflections due to lattice distortions has not been observed for $\mathrm{TiOCl}$ or $\mathrm{TiOBr}$ up to the present date..$^{2,6,9,15,18,19}$
Monoclinic symmetry $P 2_{1} / m$ (a-axis unique) has been established for the twofold superstructures of the lowtemperature phases of $\mathrm{TiOCl}$ and $\mathrm{TiOBr},{ }^{2,5}$ because it is the only symmetry for which the structural distortions are compatible with a spin-Peierls state as well as with the Raman spectrum and with two independent $\mathrm{Ti}$ atoms observed in NMR. ${ }^{2,8,20}$

The diffraction in the incommensurate phase is characterized by two modulation wave vectors,

$$
\mathbf{q}^{1}=\left(\sigma_{1}, \frac{1}{2}+\delta, 0\right), \quad \mathbf{q}^{2}=\left(-\sigma_{1}, \frac{1}{2}+\delta, 0\right),
$$

with $\sigma_{1} \approx 0.07$ and $\delta \approx 0.01$ continuously dependent on temperature. ${ }^{9}$ The modulation is two dimensional if the orthorhombic symmetry of the high-temperature structure is preserved in the incommensurate phase. Monoclinic symmetry (c-axis unique) allows a 1D modulation, whereby the two modulations of Eq. (1) arise from the two orientations of the monoclinic structure on the pseudo-orthorhombic lattice. ${ }^{9}$ Evidence for a c-axis monoclinic structure has come from electron diffraction and Raman spectroscopy. ${ }^{6,20}$

Here we present results from $\mathrm{x}$-ray diffraction experiments on $\mathrm{TiOCl}$ that give direct proof of the c-axis unique monoclinic distortion of the lattice in the incommensurate phase. The deviation from $90^{\circ}$ of the monoclinic angle $\gamma$ is found to be independent of temperature at a value of $\gamma$ $=90.023(2)^{\circ}$. This result suggests that the phase transition at $T_{c 2}$ is a first-order phase transition, contrary to the signature of this transition in the temperature dependencies of other properties. $^{1,7-9,13}$

\section{EXPERIMENT}

Single crystals of $\mathrm{TiOCl}$ were grown by gas transport. ${ }^{17}$ A single crystal of dimensions of approximately $0.1 \times 0.02 \times 0.001 \mathrm{~mm}^{3}$ was glued to a carbon fiber that was attached to a closed-cycle helium cryostat mounted on the Huber four-circle diffractometer at beamline D3 of Hasylab (DESY, Hamburg). Monochromatic x rays of wavelength $\lambda=0.5000 \AA$ were employed for the diffraction experiments at Hasylab. 
With the temperature set to $T=72.0 \mathrm{~K}$, diffraction confirmed the orthorhombic basic structure as well as the presence of satellite reflections at $\pm \mathbf{q}^{1}$ and $\pm \mathbf{q}^{2}$ [Eq. (1)]. The second component of both modulation wave vectors appeared as $\frac{1}{2}$ in all $\omega$ scans. In a first experiment, $\omega$ scans were used to measure the diffracted intensity at positions of the mixed second-order satellite reflections of the type $\mathbf{q}^{1} \pm \mathbf{q}^{2}$. The presence of mixed second-order satellite reflections would provide evidence for a 2D incommensurate modulation wave and would thus indicate orthorhombic symmetry for the intermediate phase. However, intensity was not found at these positions for 38 measured second-order satellite reflections within the range $0.3<\sin (\theta) / \lambda<0.7 \AA^{-1}$. Although the failure to observe mixed second-order satellite reflections does not provide evidence for a particular symmetry, these observations are in agreement with monoclinic symmetry (unique axis $\mathbf{a}, \mathbf{b}$, or $\mathbf{c}$ ) while it does not exclude the possibility of orthorhombic symmetry.

In a second experiment, $\omega$ scans and $2 \theta$ scans were performed at selected main reflections. Different distortions of the lattice should lead to different splittings of reflections if the crystal is twinned. As noticed in Sec. I, the relatively large width of the reflections in $\omega$ scans prevented the observation of splittings in scans of this type. $2 \theta$ scans were performed with the crystal set in diffracting orientation of selected reflections and with narrow detector slits corresponding to an acceptance angle for scattered radiation of $0.003^{\circ}$. A double maximum was found for the $(\overline{4}, 4,0)$ reflection in the incommensurate phase (Fig. 1). The temperature dependence of this reflection showed a single peak of a width determined by the resolution both above $T_{c 2}$ and below $T_{c 1}$ (Fig. 1). Small deviations from the Gaussian peak shape are visible in all scans of Fig. 1. They are probably the result of a less than perfect crystal quality. In any case, their presence at all temperatures shows this feature to be unrelated to the phase transitions. These results show that the lattice parameter $\gamma$ is different from $90^{\circ}$ in the incommensurate phase, while $\gamma=90^{\circ}$ in the room-temperature and spinPeierls phases.

$2 \theta$ scans on the $(0,3,3)$ reflection gave the same width at all temperatures. This shows that $\alpha=90^{\circ}$ within the resolution of the experiment for all three phases.

The splitting of the reflections in $2 \theta$ reflects the difference in length of the scattering vectors of the reflections $(\overline{4}, 4,0)$ and $(4,4,0)$ of a monoclinic lattice with unique axis c. The observed peak profiles are well explained by one or two Gaussian functions that have been fitted to the observed profiles in Fig. 1 as well as to profiles measured at other temperatures. The splitting of $\sim 0.020^{\circ}$ in $2 \theta$ in the incommensurate phase was used to calculate the lattice parameter $\gamma$ as a function of temperature (Fig. 2). Below $T_{c 1}$ and above $T_{c 2}$, splitting was not observed, while the width of the single peak was equal to the width of the most intense maximum in the incommensurate phase. Thus in the spin-Peierls and normal phases $\gamma=90^{\circ}$.

The varying ratio of the intensities of the two peaks in a $2 \theta$ scan is the result of small variations in the alignment of the crystal and the relative positions of different reflections, as these occur at different temperatures. In addition to the

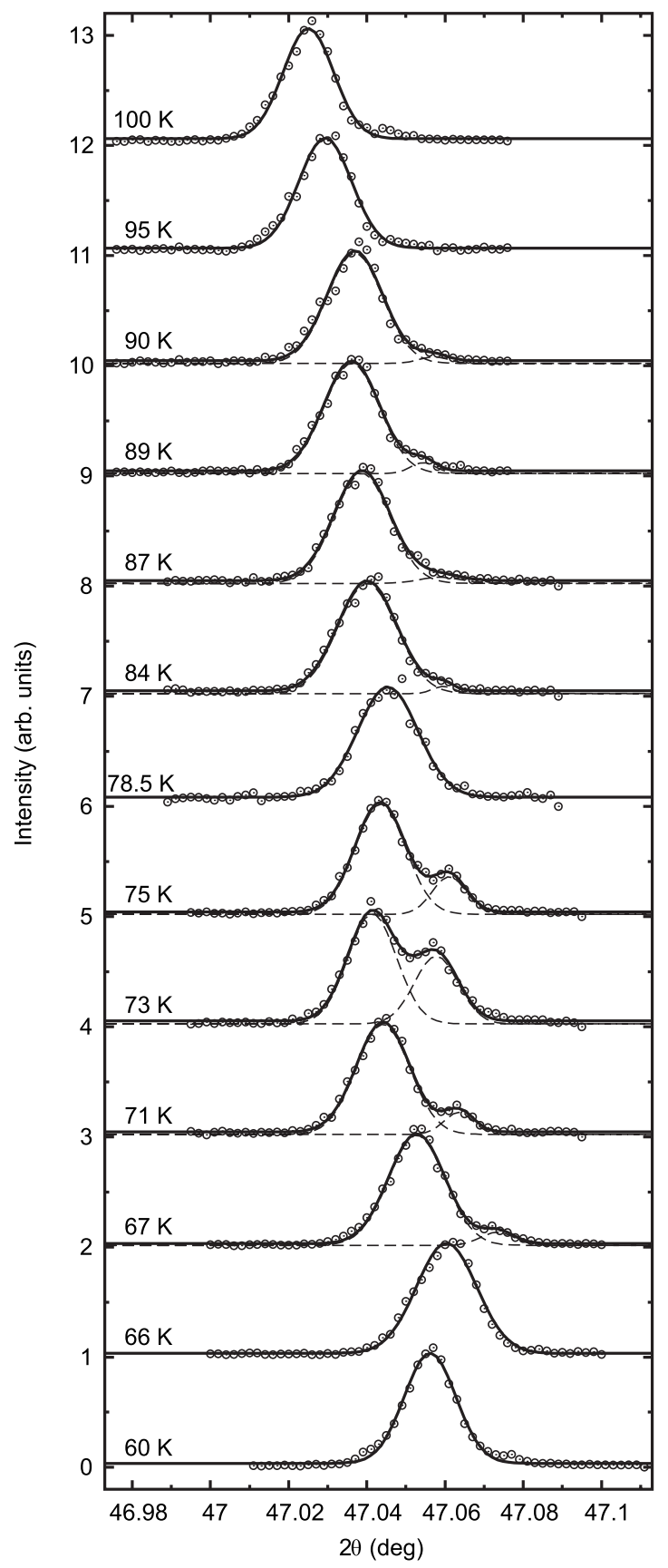

FIG. 1. $2 \theta$ Scans of the $(\overline{4}, 4,0)$ reflection at selected temperatures. Scans have been individually scaled toward a value 1 of their maximum intensities; scans at subsequent temperatures have been offset by an intensity value of 1 . Circles indicate measured values; lines denote the fit to the data with one or two Gaussian functions.

splitting in $2 \theta$, the reflection maxima may correspond to different crystal orientations (different values of $\omega$ ), because of slightly different orientations of the two domains. Apparently, the latter splitting was so large that for most measurements one of the two peaks was not entirely in scattering position. A second peak was entirely absent in the scan taken at a temperature of $78.5 \mathrm{~K}$. A splitting thus was not observed and the monoclinic angle could not be determined for this temperature. A better characterization of the diffraction could 


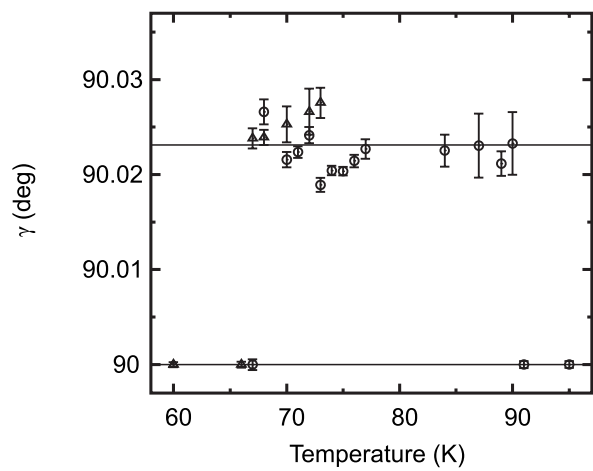

FIG. 2. Temperature dependence of the monoclinic angle $\gamma$. Horizontal lines indicate $90^{\circ}$ and the average value of the monoclinic angle of $\gamma=90.023(2)^{\circ}$. Circles were measured in the first cooling run and triangles in the second.

have been achieved by repeated $2 \theta$ scans for several values of $\omega$ on a narrow grid around the optimal calculated $\omega$. Unfortunately, sufficient beam time at the synchrotron was not available for such an elaborate experiment. Nevertheless, we believe we have observed a second peak of sufficient intensity in the majority of $2 \theta$ scans to have determined the monoclinic angle $\gamma$ over the whole range of temperatures of the incommensurate phase (Fig. 2). For these reasons, we refrain from an analysis of the intensities of the reflections in $2 \theta$ scans.

\section{DISCUSSION}

We have found that the lattice parameter $\gamma$ is different from $90^{\circ}$ in the incommensurate phase of $\mathrm{TiOCl}$, while $\gamma$ $=90^{\circ}$ in both the spin-Peierls and room-temperature phases. This result is in agreement with a c-axis unique monoclinic crystal structure in the incommensurate phase..$^{6,18,20}$ Together with the quantitative analysis of the diffracted intensities, this result allows the unique assignment of the $(3+1)$-dimensional superspace group $P 2 / n\left(\sigma_{1} \sigma_{2} 0\right) 00$ to this structure. ${ }^{18}$

The recovery of $\gamma=90^{\circ}$ below $T_{c 1}$ shows that the spinPeierls state must have a different symmetry than c-axis unique monoclinic, and the present results confirm the previously determined monoclinic space group $P 2_{1} / m$ (a-axis unique) for the twofold superstructure of this phase. ${ }^{2}$

An unexpected result of the present experiments is that the observed peak splitting is independent of temperature with a monoclinic angle of $\gamma=90.023(2)^{\circ}$ at temperatures between $T_{c 1}$ and $T_{c 2}$ (Fig. 2). This implies that both transitions are first-order phase transitions. Unambiguous experimental evidence is available for the transition at $T_{c 1}$ being a first-order phase transition. ${ }^{1}$ While there seems to have been a general agreement that the transition at $T_{c 2}$ would be a second-order phase transition, the experimental situation is less clear than for the transition at $T_{c 1}$. The temperature dependencies of several properties show but weak anomalies at $T_{c 2}$, which thus prevent the character of the phase transition at $T_{c 2}$ from being uniquely determined, or which are not even clear signatures of a phase transition. This pertains to the temperature dependencies of the signal in electron spin resonance, ${ }^{7,21}$ of the specific heat, ${ }^{13,14}$ of the magnetic susceptibility, ${ }^{1,14}$ of the thermal expansion, ${ }^{14}$ of the signal in NMR, ${ }^{8}$ of the signal in muon relaxation experiments, ${ }^{22}$ and of the diffracted intensities of incommensurate satellite reflections in X-ray diffraction. ${ }^{9,19}$ In the last case, the diffracted intensities of satellite reflections have been found to continuously grow on cooling below $T_{c 2}$ within the incommensurate phase, but with a temperature dependence that could not be accurately determined near $T_{c 2}$. Thus a small jump of these intensities at $T_{c 2}$ cannot be excluded on the basis of the available experimental information.

The occurrence of an incommensurate intermediate phase has previously been ascribed to frustration between spinPeierls pair formation and interchain interactions, and it was pointed out that the spin-Peierls state is intrinsically frustrated by elastic Ti-O-Ti coupling between chains. ${ }^{14,18,19}$ In order to reconcile this model with the present observations, one might, for example, suppose that the incommensurate satellite reflections appear with a finite intensity at $T_{c 2}$, thus being in agreement with the first-order character of the phase transition. However, this scenario does not provide an explanation for the different temperature dependencies of the magnitudes of the incommensurate distortion and the monoclinic splitting. 9,19

Instead, we believe that a second kind of attractive interaction is necessary in order to explain the different temperature dependencies of the incommensurate modulation and the c-axis monoclinic lattice distortion in the incommensurate phase. The most likely candidate for the second type of interaction is antiferromagnetic (AF) coupling between Ti ions on neighboring ribbons [i.e., between Ti atoms with positions differing by approximately $( \pm 0.5, \pm 0.5,0.25)]$. This idea is supported by the results of recent x-ray absorption and photoemission experiments and cluster calculations that have indicated interchain superexchange interactions, due to the hybridization of the magnetic $3 d$ electron of Ti with oxygen $2 p$ orbitals, of a magnitude of $12 \%$ of the magnitude of the intrachain exchange interactions. ${ }^{23}$

We propose that at $T_{c 2}$ a state of antiferromagnetic order develops that is governed by intrachain and interchain antiferromagnetic interactions between the magnetic moments on the $\mathrm{Ti}$ atoms. The antiferromagnetic interactions on the layers of Ti-O parallel to the $\mathbf{a}, \mathbf{b}$ plane represent frustrated $2 \mathrm{D}$ interactions on the orthorhombic lattice. The observed monoclinic distortion of the lattice then is responsible for the fact that the free energy of the material is lowered by this type of interaction, and it thus explains the first-order character of the transition at $T_{c 2}$. The true nature of the magnetic order cannot be determined on the basis of the present experiment. But it seems that the observed temperature dependence of the magnetic susceptibility ${ }^{1}$ is in agreement with long-range magnetic order with a temperature-dependent magnitude of the fluctuations of the directions of the magnetic moments about the direction of perfect AF alignment.

Atomic displacements related to antiferromagnetic order usually are zero or they have a small magnitude that cannot be detected in ordinary (synchrotron-radiation) x-ray diffraction experiments. Therefore, the origin of the incommensurate satellite reflections cannot be the proposed antiferromag- 
netic order on the Ti-O plane, but should rather be ascribed to the formation of a spin-Peierls-like distortion, albeit frustrated by elastic Ti-O-Ti interactions between neighboring ribbons and by the competition between the formation of local spin-singlet pairs and long-range antiferromagnetic order of the spins of the Ti atoms. This frustration is responsible for the incommensurate character of the modulation between $T_{c 2}$ and $T_{c 1}$. Structure refinements have shown that the incommensurate atomic displacements can indeed be considered as an incommensurate version of the spin-Peierls distortion. ${ }^{9,18}$

Both long-range antiferromagnetic order and the spinPeierls state rely on an antiferromagnetic coupling between neighboring spins. The unique feature of spin-Peierls type order is that the structural distortion together with the lowdimensional character of the magnetic interactions lead to a situation where each spin is strongly coupled to one neighboring spin, while the other magnetic couplings are weaker. This induces the formation of a singlet pair by these two spins that is not coupled magnetically to the remainder of the lattice. The spin-Peierls state thus is commensurate by definition-as has been found for the twofold superstructure of the low-temperature phase. ${ }^{2,5}$

The incommensurate modulation has been described by a sinusoidal displacement wave of the atomic positions. ${ }^{9}{ }^{918}$ The effect is that along the ribbons the structure varies between $\mathrm{Ti}-\mathrm{Ti}$ dimers, a regular array of $\mathrm{Ti}$ atoms, and anything in between. ${ }^{9}$ One may thus assume a threshold for the Ti-Ti distance beyond which the modulation of the magnetic interactions is sufficiently strong to induce the formation of a spin-singlet pair; this pair of electrons is then no longer available for the delocalized state of long-range antiferromagnetic order. The number of such pairs increases with decreasing temperature, because of the increasing magnitude of the modulation, until too many electrons have been removed from the antiferromagnetic state for it to be stable, and the crystal transforms into the spin-Peierls state. On the other hand, it is known that a set of weak first-order satellite reflections cannot distinguish between modulation waves of different shapes. An equivalent assumption thus is that the modulation wave will have a shape that implies that a fraction of the spins form perfect spin-Peierls dimers, with an increasing number of these dimers on decreasing temperature.

The coincidence of the transition temperatures for the development of antiferromagnetic order and the onset of frustrated spin-Peierls distortion may be related to the restric- tions imposed by symmetry. Both long-range antiferromagnetic order and the formation of coherent spinPeierls dimers are forbidden within orthorhombic symmetry. The lowering of the lattice symmetry toward c-axis monoclinic then allows both types of interaction to induce longrange ordered structures. Accordingly, it cannot be excluded that there is a small jump in intensities of the satellite reflections at $T_{c 2}$, reflecting a jump of the incommensurate spinPeierls order parameter from zero to a finite value at this temperature. Apparently, the formation of spin-Peierls dimers is highly frustrated on the c-axis monoclinic lattice, while complete spin-Peierls order prevails once the symmetry is changed to a-axis monoclinic.

\section{CONCLUSIONS}

We have performed temperature-dependent $\mathrm{x}$-ray diffraction experiments on $\mathrm{TiOCl}$ that indicate a first-order character of the phase transition at $T_{c 2}$. This result is reconciled with previous experiments, which have indicated a continuous character of the transition at $T_{c 2}$, by the following scenario.

The phase transition at $T_{c 2}$ is the result of the development of antiferromagnetic order of the spins of the $\mathrm{Ti}$ ions, which is facilitated by the c-axis monoclinic distortion of the crystal lattice. The lowering of the lattice symmetry also allows the development of incommensurate, frustrated spinPeierls modulations of the atoms. The amplitude of this wave grows on cooling, and thus provides the continuous order parameter as observed in various experiments. A growing amplitude may actually imply that the number of spin-singlet pairs grows on cooling, thus removing an increasing number of electrons from the antiferromagnetic state.

At $T_{c 1}$ too many electrons have been removed for the antiferromagnetic state to be stable, and the crystal transforms to the spin-Peierls state that possesses a-axis monoclinic symmetry. Antiferromagnetic order is lost, because the antiferromagnetic interchain interactions are again perfectly frustrated on the a-axis monoclinic lattice.

\section{ACKNOWLEDGMENTS}

Single crystals were grown by A. Suttner. W. Morgenroth is thanked for assistance with the synchrotron experiment. Diffraction experiments with synchrotron radiation were performed at beamline D3 of Hasylab, DESY, Hamburg, Germany. Financial support has been obtained from the German Science Foundation (DFG). *smash@uni-bayreuth.de

${ }^{1}$ A. Seidel, C. A. Marianetti, F. C. Chou, G. Ceder, and P. A. Lee, Phys. Rev. B 67, 020405(R) (2003).

${ }^{2}$ M. Shaz, S. van Smaalen, L. Palatinus, M. Hoinkis, M. Klemm, S. Horn, and R. Claessen, Phys. Rev. B 71, 100405(R) (2005).

${ }^{3}$ T. Sasaki, M. Mizumaki, K. Kato, Y. Watabe, Y. Nishihata, M. Takata, and J. Akimitsu, J. Phys. Soc. Jpn. 74, 2185 (2005).

${ }^{4}$ M. Hoinkis, M. Sing, J. Schäfer, M. Klemm, S. Horn, H.
Benthien, E. Jeckelmann, T. Saha-Dasgupta, L. Pisani, R. Valenti, and R. Claessen, Phys. Rev. B 72, 125127 (2005).

${ }^{5}$ L. Palatinus, A. Schönleber, and S. van Smaalen, Acta Crystallogr., Sect. C: Cryst. Struct. Commun. 61, i47 (2005).

${ }^{6}$ T. Sasaki, M. Mizumaki, T. Nagai, T. Asaka, K. Kato, M. Takata, Y. Matsui, and J. Akimitsu, arXiv:cond-mat/0509358 (unpublished).

${ }^{7}$ V. Kataev, J. Baier, A. Moller, L. Jongen, G. Meyer, and A. 
Freimuth, Phys. Rev. B 68, 140405(R) (2003).

${ }^{8}$ T. Imai and F. C. Chou, arXiv:cond-mat/0301425 (unpublished).

${ }^{9}$ S. van Smaalen, L. Palatinus, and A. Schönleber, Phys. Rev. B 72, 020105(R) (2005).

${ }^{10}$ G. Caimi, L. Degiorgi, P. Lemmens, and F. C. Chou, J. Phys.: Condens. Matter 16, 5583 (2004).

${ }^{11}$ G. Caimi, L. Degiorgi, N. N. Kovaleva, P. Lemmens, and F. C. Chou, Phys. Rev. B 69, 125108 (2004).

${ }^{12}$ P. Lemmens, K. Y. Choi, G. Caimi, L. Degiorgi, N. N. Kovaleva, A. Seidel, and F. C. Chou, Phys. Rev. B 70, 134429 (2004).

${ }^{13}$ J. Hemberger, M. Hoinkis, M. Klemm, M. Sing, R. Claessen, S. Horn, and A. Loidl, Phys. Rev. B 72, 012420 (2005).

${ }^{14}$ R. Rückamp, J. Baier, M. Kriener, M. W. Haverkort, T. Lorenz, G. S. Uhrig, L. Jongen, A. Möller, G. Meyer, and M. Grüninger, Phys. Rev. Lett. 95, 097203 (2005).

${ }^{15}$ C. Kato, Y. Kobayashi, and M. Sato, J. Phys. Soc. Jpn. 74, 473 (2005).

${ }^{16}$ P. Lemmens, K. Choi, R. Valenti, T. Saha-Dasgupta, E. Abel, Y. Lee, and F. Chou, New J. Phys. 7, 74 (2005).

${ }^{17}$ H. Schäfer, F. Wartenpfuhl, and E. Weise, Z. Anorg. Allg. Chem.
295, 268 (1958).

${ }^{18}$ A. Schönleber, S. van Smaalen, and L. Palatinus, Phys. Rev. B 73, 214410 (2006)

${ }^{19}$ A. Krimmel, J. Strempfer, B. Bohnenbuck, B. Keimer, M. Hoinkis, M. Klemm, S. Horn, A. Loidl, M. Sing, R. Claessen, and M. v. Zimmermann, Phys. Rev. B 73, 172413 (2006).

${ }^{20}$ D. Fausti, T. T. A. Lummen, C. Angelescu, R. Macovez, J. Luzon, R. Broer, P. Rudolf, P. H. M. van Loosdrecht, N. Tristan, B. Buchner, S. van Smaalen, A. Moller, G. Meyer, and T. Taetz, Phys. Rev. B 75, 245114 (2007).

${ }^{21}$ D. V. Zakharov, J. Deisenhofer, H. A. Krug von Nidda, P. Lunkenheimer, J. Hemberger, M. Hoinkis, M. Klemm, M. Sing, R. Claessen, M. V. Eremin, S. Horn, and A. Loidl, Phys. Rev. B 73, 094452 (2006).

${ }^{22}$ P. J. Baker, S. J. Blundell, F. L. Pratt, T. Lancaster, M. L. Brooks, W. Hayes, M. Isobe, Y. Ueda, M. Hoinkis, M. Sing, M. Klemm, S. Horn, and R. Claessen, Phys. Rev. B 75, 094404 (2007).

${ }^{23}$ R. Macovez, J. Luzon, J. Schiessling, A. Sadoc, L. Kjeldgaard, S. van Smaalen, D. Fausti, P. H. M. van Loosdrecht, R. Broer, and P. Rudolf, Phys. Rev. B 76, 205111 (2007). 